\title{
Moral Experience of Canadian Healthcare Professionals in Humanitarian Work
}

\author{
Matthew R. Hunt, PhD
}

Department of Clinical Epidemiology and Biostatistics, McMaster University, Hamilton, Ontario Canada

\author{
Correspondence: \\ Matthew R. Hunt, PhD \\ Department of Clinical Epidemiology and \\ Biostatistics \\ McMaster University \\ 1200 Main Street West \\ HSC 3V43B \\ Hamilton, Ontario, Canada L8N $3 Z 5$ \\ E-mail: 1matthew.hunt@gmail.com
}

\begin{abstract}
An earlier version of the article was included in the author's doctoral dissertation in the Division of Experimental Medicine, McGill University, Montreal, Quebec Canada.
\end{abstract}

The author is supported by a post-doctoral fellowship from the Canadian Institutes of Health Research.

Keywords: emergency; ethics; healthcare professional; humanitarian; moral experience; natural hazard; non-governmental organization

Abbreviations:

$\mathrm{HCP}=$ healthcare provider

$\mathrm{NGO}=$ non-governmental organization

Received: 10 January 2009

Accepted: 17 February 2009

Web publication: 09 January 2010

\section{Abstract}

Introduction: Expatriate healthcare professionals frequently participate in international relief operations that are initiated in response to disasters due to natural hazards or humanitarian emergencies in low resource settings. This practice environment is significantly different from the healthcare delivery environment in the home countries of expatriate healthcare professionals. Human rights, public health, medicine, and ethics intersect in distinct ways as healthcare professionals provide care and services in communities affected by crisis. Purpose: The purpose of this study was to explore the moral experience of Canadian healthcare professionals during humanitarian relief work.

Methods: This is a qualitative study with 18 semi-structured individual interviews based on Interpretive Description methodology. There are two groups of participants: (1) 15 healthcare professionals (nine doctors, five nurses, and one midwife) with more than three months experience in humanitarian work; and (2) three individuals who have experience as human resource or field coordination officers for humanitarian, non-governmental organizations. Participants were recruited by contacting non-governmental organizations, advertisement at the global health interest group of a national medical society, word of mouth, and a snowball sampling approach in which participants identified healthcare professionals with experience practicing in humanitarian settings who might be interested in the research.

Results: Five central themes were identified during the analysis: (1) examination of motivations and expectations; (2) the relational nature of humanitarian work; (3) attending to steep power imbalances; (4) acknowledging and confronting the limits of what is possible in a particular setting; and (5) recognition of how organizational forms and structures shape everyday moral experience. Discussion: Humanitarian relief work is a morally complex activity. Healthcare professionals who participate in humanitarian relief activities, or who are contemplating embarking on a humanitarian project, will benefit from carefully considering the moral dimensions of this work. Humanitarian organizations should address the moral experiences of healthcare professionals in staff recruitment, as they implement training prior to departure, and in supporting healthcare professionals in the field.

Hunt MR: Moral experience of Canadian healthcare professionals in humanitarian work. Prehosp Disaster Med 2009;24(6):518-524.

\section{Introduction}

Humanitarian relief work entails important shifts of professional, regulatory, and cultural environments for expatriate healthcare professionals (HCPs). Humanitarian work frequently occurs in contexts of political and social instability. ${ }^{1}$ Populations that require emergency assistance may have been displaced from their homes. ${ }^{2}$ Humanitarian crises often prompt the response of dozens of governmental and non-governmental organizations (NGOs), making coherent and efficient program development difficult. ${ }^{3}$ Available supplies, infrastructure and trained personnel frequently are insufficient to address the needs of the population. ${ }^{4}$ Population health considerations are a constant con- 
cern. ${ }^{1}$ In some settings, the need to address security and protection for local populations and expatriate workers is acute. ${ }^{6}$ Cultural and linguistic barriers exist between expatriate $\mathrm{HCPs}$ and the local population. ${ }^{6}$ These features of healthcare practice in humanitarian crises present clinical, logistical, and ethical challenges for HCPs.

There is broad recognition that humanitarian assistance is a morally complex activity, and there is potential for humanitarian activities to result in unintended harm. This awareness has led to initiatives designed to provide standards of best practice and increase the accountability of aid organizations. ${ }^{7-9}$ During the past two decades, humanitarian practitioners, donors, policy-makers, and academics have engaged in considerable discussion and debate regarding the ethics of humanitarian action. ${ }^{10-12}$ The detailed analysis of ethics in humanitarian assistance has yet to be extended to all aspects of these interventions. To date, there has been less discussion concerning the ethics of healthcare practice for expatriate $\mathrm{HCPs}$ who participate in humanitarian missions. Healthcare practitioners in humanitarian work frequently experience ethically complex situations. ${ }^{13-15}$ Expatriate HCPs may question how to best address the ethics of healthcare practice in contexts that are significantly different from their "ordinary" practice environment in their home country.

\section{Methods}

This inquiry is guided by the following question: "What is the moral experience of HCPs during humanitarian work?" The operational definition of "moral experience" is:

\section{Moral experience comprises, but is not limited to, aspects of experience that give rise to sentiments of 'right and wrong', 'good or bad', remorse, guilt, responsibility, justice, or distressed or fulfilled conscience. Moral experience goes beyond moral justification and reasoning, and is rooted in social and cultural contexts and relationships. ${ }^{16}$}

The methodological framework employed in this study is Interpretive Description. ${ }^{17,18}$ Interpretive Description is a qualitative research methodology that draws upon elements of Grounded Theory and Naturalistic Inquiry. ${ }^{19,20}$ Interpretive Description uses inductively derived thematic analysis to identify patterns among and between individual cases within a phenomenon of interest.

\section{Data Collection}

In-depth interviews using open-ended questions were conducted following an interview guide. A purposive sampling strategy was used to recruit a diverse set of participants by several means including contacting NGOs, advertisement at the global health interest group of a national medical society, word of mouth, and a snowball sampling approach in which participants suggested others who might be interested in taking part in the research. Interviews were audio-recorded and transcribed. Fourteen women and four men participated in the study.

There were two groups of participants. The first group included 15 HCPs (nine physicians, five nurses, and one mid- wife). Each participant had spent at least three months as an $\mathrm{HCP}$ in humanitarian work. The participants worked in a range of settings including hospitals, rural health clinics, mobile clinics, and refugee camps. Their responsibilities included: clinical care, supervision of local HCPs, public health programming, oversight of health clinics, human rights work, program evaluation, needs assessment, and logistical support of displaced persons during humanitarian crises.

The second group included three human resource and field coordination officers of international NGOs. These participants had responsibility for the training, recruitment or support of HCPs. Each of these participants had experience with a different NGO. The rationale for including this component of the inquiry was to access organizational perspectives regarding the ethical dimensions of humanitarian health practice.

The secondary data source consisted of documentary material including narrative articles in medical journals relating to participation of expatriate $\mathrm{HCPs}_{\mathrm{s}}$ in global health projects, as well as blogs and published essays in which HCPs discuss their experiences in humanitarian work.

\section{Data Processing}

Processing of the data acquired was initiated as each transcript was completed so that insights developed during early interviews could be incorporated into the ongoing data collection. Initial coding helped to refine the process of participant selection and inform theoretical sampling. Preliminary impressions and early categories were tested during later interviews. Coding was intended to identify categories and linkages in the data, and to explore relationships and patterns. Thematic analysis was used to illuminate the phenomenon of moral experience of HCPs in humanitarian work.

An overview of the provisional findings was provided to the participants. Participants were invited to respond to this overview and discuss how consistent the findings were with their own experiences. This feedback was used to refine the analysis. Ten of 18 participants participated in these 'member checks'. The documentary sources also were used to evaluate the provisional findings that had been generated based on analysis of the interview transcripts. The documentary materials were compared to the provisional findings by asking the following question: "How do the documentary sources reflect the moral experience of HCPs in ways that are consistent with, or different from, the provisional findings?" The goal of the member checks and comparison with the documentary materials was to confirm or challenge the analytic structure that was developed.

\section{Results}

A series of five interconnected themes were identified: (1) examining motivations and expectations; (2) the relationality of humanitarian action; (3) attending to steep imbalances of power in humanitarian work; (4) acknowledging and confronting limits to what can be accomplished; and (5) recognizing how organizational forms and structures shape everyday moral experience. Selected verbatim quotes are included to illustrate aspects of the analysis. 
"The Overriding 'Why' Question": Examining Motivations and Expectations

Motivations play a key role in framing the moral experience of HCPs. A participant described motivation as the "overriding 'why' question" and necessary starting point to consider the moral dimensions of humanitarian work. The participants view motivations as influencing how $\mathrm{HCPs}$ act in the field and how they experience challenging situations. Many participants acknowledged a cluster of motivations that influenced their decision to participate in humanitarian work. A primary motivation for all participants was the desire to offer tangible assistance to people in need. Some interviewees felt that having skills as a clinician provided them the possibility of helping in a direct fashion:

\section{I am a health professional that has some skills and} knowledge that, you know, benefit a lot more people by me going over there for six months than continuing to go down to my job.

Many participants also emphasized ways that they benefited from engaging in humanitarian work. Participants described motivations to learn about a new part of the world, have new experiences, challenge oneself, or develop relationships with people from other cultures. Participants expressed that it was healthy to have such goals: "A lot of people who do it... in the end who stick it out, they do it for their own self-fulfillment as much as do it to save people, and I think that's a reality." In contrast, several participants discussed motivations aimed to "save the world". These participants were deliberate in distancing themselves from this idea. One concern was that such a view creates a "really unhealthy dynamic" and casts the expatriate HCP in the role of rescuer and the beneficiaries of their assistance as passive recipients.

Motivation also directly influences how HCPs interpret their experiences. Participants expressed that certain motivations increase susceptibility to feelings of disillusionment or anxiety. Overly idealized motivations can lead to heightened self-doubt and decreased resiliency:

... you don't always see the results and you bave these questions, "is this really worth it?" Then you start to think, "Well, what am I doing here? I have sacrificed so much to come bere, and it is not clear that I am making any headway."

Unrealistic expectations can lead to disappointment, and some HCPs may quit and withdraw from the work. Other motivations help sustain $\mathrm{HCPs}_{\mathrm{s}}$ in difficult circumstances. One participant related how she helps prepare HCPs for their first international assignments. A message that she tries to convey in these training sessions is:

No matter what you run into, whether it's fear, if you're in a war zone, whether it's an entanglement with your bousemate or you are struggling with... what you are witnessing, I said not to lose perspective of what brought you bere.
Motivations influence how humanitarians act in the field and how they relate to colleagues and beneficiaries. Several participants emphasized that HCPs should evaluate, and challenge their own motivations on an ongoing basis. Motivations will change over time and HCPs should reflect on the evolution of their goals and expectations.

\section{"Journeying Alongside": The Relational Nature of Humanitarian Work}

Relationships are central to the moral experience of HCPs in humanitarian work. Relationships have the potential to be sites of ethical concern for participants; they also are key sources of motivation and satisfaction. Four aspects of the relationality of humanitarian work are the inter-cultural nature of many of these relationships, the challenge of making space for collaboration and dialogue in emergency settings, the importance of team relationships, and the ways that relationships provide grounding for humanitarian action.

In humanitarian settings, intercultural relationships among HCPs, national staff, and community members are the norm. The participants worked within NGO teams that were composed of individuals who came from different nations, and were multicultural. In this context, attending to culture is critical. Participants noted that expatriate HCPs should endeavor to learn about the local culture and be reflective regarding their own cultural frameworks. A participant expressed: "there are all these cultural norms that I need to know before my role can ever really be established." Participants described challenges in working across cultural frameworks, interacting with traditional health practitioners, and understanding local practices related to health.

The participants valued collaborative approaches in humanitarian work. Interviewees acknowledged that there often are important obstacles to the creation of collaborative relationships between international agencies and others. While in many situations, it may be efficient in the short term to provide interventions separate from local actors, several participants underlined the added benefit of promoting a participatory approach. These participants expressed frustration with colleagues who did not promote collaboration or who lacked communication skills. A participant noted that to be effective in this work, HCPs need strong interpersonal abilities and even "common sense, political negotiation skills." Collaboration is ultimately dependent on developing "trust relationships" that require time and care to develop.

The participants worked closely with local and expatriate colleagues. Expatriate HCPs can derive significant support and encouragement from their close colleagues in the field. As one participant described, team members become "brothers and sisters." He noted that "you depend on them for everything" and that because of the strong bonds that are developed, he would "lie down in traffic for them". Participants noted the value of being part of an integrated and supportive team. In contrast, HCPs who enter humanitarian work and think that they can make a difference "all on their own", face a major pitfall. A divided or distrustful team will be less effective and contribute to disappointment and frustration.

Many participants ground humanitarian action in terms of relationships. Several participants noted that the context 
of humanitarian crises can diminish the dignity of recipients of assistance. The urgency and volume of health needs, as well as cultural and linguistic barriers, challenge $H C P s$ as they seek to respond to this situation. Even in settings of acute need, participants advocated for ensuring that the stories of beneficiaries were heard and that the attention and gaze of humanitarian practitioners rests on individuals, as well as whole communities and populations. Many participants also emphasized the importance of solidarity between themselves and local people living in settings affected by armed conflict or disaster due to natural hazards. Participants described a primary focus of their work in regards to their shared humanity with beneficiaries of aid. Participants spoke of "accompanying", "journeying alongside", "meeting as human beings" or having "solidarity" with the local community. In this way, participants emphasized mutuality and interconnections between those who provide and those who receive assistance.

\section{Attending to Steep Imbalances of Power}

Asymmetrical power relationships are inherent in healthcare interactions. These power differentials are amplified in the context of healthcare practice in humanitarian settings. Participants frequently spoke about the centrality of power:

\section{And on the topic of power we need to really educate ourselves and our colleagues going to the field really about how much power we have: over priorities, over programs, over our staff... and the power that we hold over the beneficiaries.}

In many cases, the participants were explicit about the importance and prevalence of asymmetrical power relations. A participant described that: "the power differentials are unbelievable, I think it is fair to say. The sheer power that we have. I think that we should recognize that and we need to recognize it." Power is experienced in both clinical and everyday settings. Power differentials exist even in seemingly mundane situations.

A particular concern related to how humanitarian organizations and practitioners may enact changes to healthcare practice without taking time to gain an appreciation for the local reality, or enter into dialogue with community representatives or local HCPs. A participant described the importance of listening to community members' views and concerns: "So I think, in the end, it is a lot about listening, and a lot about your feet hit the ground and just the triangulation thing of observation and listening." Several participants noted that humility is an essential attitude to avoid inappropriately imposing outside views on the local community. Several participants situated their work in the colonial history of North-South relations. They expressed concerns regarding the possibility for humanitarian activities to replicate colonial patterns.

Participants also identified situations or practices that they should seek to influence or change. In general, deciding whether to intervene involved significant reflection and discussion. These situations included issues of inadequate training or areas of healthcare services regarded as less effective. In such circumstances, participants advocated strongly for an approach that was built around education for local staff. Some social issues and practices, such as domestic violence, the position of women in society, and discrimination against a minority group, were concerns for participants, and they described efforts to discuss these issues with colleagues, community leaders, or in the context of community health teaching. A participant advised that to address such practices, "You have to be very patient, and careful, and respectful..."

Healthcare providers also feel powerless in some situations. Particularly troubling are situations in which assistance to needy communities is impeded by local powers, such as armed groups or the military, bureaucratic structures that delay the implementation of programs, or corrupt officials. Though HCPs are in positions of relative power, they also experience feelings of helplessness when they are prevented from responding to local needs.

Acknowledging and Confronting the Limits of What is Possible The participants discussed the complexity of providing health care during and after humanitarian crises. As noted by a participant, "The needs are so great and they are on so many different levels." Projects often take place in settings in which the local health system and infrastructure have been destabilized or overwhelmed. The ability to provide care and services is influenced by the availability of healthcare resources and, in some settings, other needs such as clean water, security, adequate food, and shelter. As a participant described in regards to the provision of HIV/AIDS drugs, there are more basic questions than whether medication is available; you also need "access to clean drinking water to wash down your antiretrovirals." Several participants emphasized the broader concerns of how global inequities and political structures contribute to the vulnerability of many communities affected by crises.

The participants described how uncertainty in humanitarian settings affects the provision of care. For instance, a decision to initiate tuberculosis treatment must be evaluated relative to the risk of a renewal of hostilities in the region and interruption of the treatment regime. In this context, population health concerns abound. As a result, public health considerations may influence treatment options for individual patients. Adapting to this reality can be a particular challenge for $\mathrm{HCPs}$ who do not have training or experience in public health.

Healthcare providers work within-and push againstthe constraints that are inherent in humanitarian assistance. The context of humanitarian work presents challenges that need to be overcome, or in other cases, adapted to and accepted. For some participants, the level of care that they were able to provide was less than they had anticipated: "So it was frustrating sort of coming to grips with really, really lowering expectations." Participants described how, as HCPs, they strive to maximize the relevance and effectiveness of the assistance they provide to individual patients and to communities. Several participants underlined their view that at a fundamental level, the standard of care does not alter between high-tech western healthcare settings to those of a developing country experiencing a humanitarian crisis. In both settings, the best care is provided to the most people in light of the equipment, supplies, and personnel 
that are available locally. Participants emphasized the need to do the best possible within situational limitations. However, in many circumstances, recognition of the inability to more fully address the needs of patients and communities was a source of frustration and regret for participants. Participants described how, on an individual level, they needed to focus their energy on situations that they could improve. One participant reported telling herself in reflecting on a problem that seemed beyond her ability to influence: "There really is nothing I can do about that at this time. And I am here only for a short time, so I should focus on what I can do." The ability to make an effective contribution to a humanitarian project is closely connected to having appropriate skills and capacities. This work requires more than being "a warm body with a degree in your hand". The participants discussed the varied skill set required. Several participants who had only a single humanitarian experience described going through a period of doubt concerning their ability to contribute-and questioning their suitability for the work they were assigned. A participant related the importance of having relevant knowledge or training in tropical diseases and the conditions that were most common in the project setting: “... I think people have to be really, I wish I had been, much better prepared clinically. I was going, I was a medical person going into a medical facility. I wish I had been more prepared about what I was going to be seeing and doing."

\section{Recognizing How Organizational Forms and Structures Shape} Everyday Moral Experience

Healthcare providers who participate in humanitarian work usually are members of non-governmental or inter-governmental organizations. They also become members of the wider humanitarian community. Organizational structures and norms contribute to shaping the everyday moral experience of participants. Certain organizational features are seen as problematic and contestable; other organizational features provide clarity and benefit when responding to morally complex issues.

Humanitarian organizations operating in regions of instability have tightly scripted security policies that govern the actions and movement of their personnel. These policies are aimed to promote the security of staff and projects. The restrictiveness of these regulations may be unanticipated by expatriates deployed to the field. Typically, these protocols create boundaries between expatriate and national workers, and place restrictions on the freedom of movement of expatriate personnel and the use of project vehicles. Participants acknowledged how a security incident can imperil an entire mission and the provision of care for a whole population.

However, several participants expressed concern about how such rules, though important, can impede the development of relationships with local staff or community members, and in certain situations can make it more difficult to provide care to patients. These participants described how security protocols prevented them from visiting local colleagues in their homes and created barriers to using project vehicles to evacuate patients after dark.

Clarity of project parameters was a concern for participants. A participant arrived in the field only to discover that her role and responsibilities were not clearly understood or defined:
There was nobody to train me in the field and I just bad to figure it out and that was stressful... At first I would wake up and think, "What, what am I supposed to do today?" Nobody really knew what my role was.

Role and responsibility ambiguity contribute to uncertainty and stress for HCPs. One concern related to clarity and effectiveness relates to leadership structures. In situations in which leadership responsibility was unclear, positions were unfilled, or leaders lacked necessary skills, ethical concerns were amplified. A participant who has a broad range of experiences in humanitarian work described the importance of having a clear decision-making structure and definition of roles:

\section{If you are not clear on who is actually making the end decision it is going to fall apart because there are so many extraneous variables when you are in those situations, even in a relatively calm setting...}

Participants also expressed concern for the ways that humanitarian action could result in unintended harms, or when inefficiency or confusion diminished the effectiveness of the assistance that was provided. Some humanitarian interventions become "a big machine moving through" a region. The participants described different ways that humanitarian activities can result in destabilization. Hiring local HCPs at higher wages than the Ministry of Health is able to pay can create a movement of HCPs from government institutions to non-governmental organizations. This movement also can take health workers away from more general or primary care to specialized (and better funded) programs. Participants also were concerned when available resources were not used to maximum effect, or with adequate efficiency. In particular, participants worried about inefficiency within NGOs, such as too much money being spent on consultants, or duplication and redundancy between the activities of different NGOs.

\section{Discussion}

Numerous aspects of healthcare practice in humanitarian work appear to be heavily freighted with moral considerations for HCPs. Five themes were identified in this inquiry: (1) the importance of examining motivations and expectations; (2) the relational nature of humanitarian work; (3) addressing steep imbalances of power; (4) acknowledging and confronting the limits of what is possible; and (5) recognizing how organizational forms and structures shape the moral experience of HCPs. There has been limited discussion in the literature relating to how HCPs experience the moral aspects of humanitarian work. The findings of this study contribute to illuminating this important topic.

The rationale for focusing upon "moral experience", as opposed to ethical issues or challenges, was to encourage a broad discussion of the ethical dimensions of HCP experiences in this practice context. This expanded scope was readily achieved. Local concerns or discrete incidents were consistently brought back to larger trends and patterns by the interviewees. Participants emphasized relational and contextual features of specific situations. Indeed, many par- 
ticipants raised broad concerns of global health and justice. However, this breadth also posed challenges in establishing boundaries for the inquiry. This challenge may have been particularly acute due to the diversity of the participants' experiences in humanitarian work.

The research findings point to key aspects of the moral experience of HCPs in humanitarian work. It is of interest that the identified themes apply to many other settings of clinical practice. However, the manner in which these are manifest is distinctive in relief initiatives. In particular, humanitarian settings may amplify and extend 'ordinary' moral considerations. One aspect is the seemingly totalizing nature of this work environment. Healthcare providers in humanitarian work do not work set hours or specific shifts and then step back into their everyday lives. In humanitarian work, boundaries between work and nonwork tend to dissolve. Family and friends are geographically distant. In consequence, there are fewer opportunities to separate from a situation, to get a different perspective, or to reorient. Humanitarian work often is characterized by rapid change and volatility. This is true particularly in acute emergency settings. This work also can be characterized by routine and even tedium. Participants described the variety among these experiences. The social world of local projects often is very circumscribed. Relationships with colleagues are of vital importance for $\mathrm{HCPs}$, and the local project team becomes the primary reference point for HCPs. ${ }^{21}$ These features contribute to shaping the particular moral and social world of humanitarian health practice for expatriate HCPs.

Various considerations support the need for HCPs and NGOs to carefully consider the moral dimensions of this work. Addressing moral experience will help support the well-being of HCPs. Some aspects of moral experience identified in this research are linked to feelings of disillusionment, frustration, or uncertainty for HCPs. These factors can influence the care and services provided to communities and individuals who receive assistance during crises. The findings also suggest aspects of organizational structure and practice that influence the moral experience of expatriate HCPs. Thus, the study findings can help focus attention on moral dimensions of this domain of healthcare practice. Several considerations for expatriate HCPs and humanitarian organizations can be highlighted.

First, $\mathrm{HCP}_{\mathrm{s}}$ can examine their motivations and expectations for participating in humanitarian work. Having a cluster of motivations informed by both altruistic and personal goals appears to be beneficial, as well as having expectations that are realistic. Frank discussion of motivations and expectations with an experienced humanitarian practitioner could help to clarify these issues for HCPs who are considering participation in humanitarian work. Experienced $\mathrm{HCPs}$ also may benefit from periodically revisiting motivations and expectations as these will evolve and change over time.

Second, this study suggests approaches that can promote the provision of care adapted to local needs and sensitive to local concerns. Specifically, approaching humanitarian work with humility, awareness of asymmetries of power, and an orientation towards building collaboration will foster attentiveness to local needs and concerns. Such an approach is consistent with values of solidarity and respect for persons.
As experienced by the participants, there may be situations when local values and perspectives appear to clash with the moral commitments and expectations of expatriate HCPs. Healthcare providers will benefit from opportunities to discuss and evaluate such scenarios with colleagues, and will be assisted by clear organizational policies and processes to orient the response to such issues. Healthcare providers also can reflect on how they support and promote the dignity of individuals who receive assistance and care. There are aspects of humanitarian crises and the relief operations that ensue, that can diminish the dignity of beneficiaries. Efforts on the part of HCPs to embody solidarity and mutuality, and an acknowledgement of interconnections, can promote the dignity of individuals affected by disaster or conflict.

Third, HCPs who are considering participation in a humanitarian project should assess their level of preparation and suitability for this work. This evaluation may help guide HCPs towards accessing technical or language training, cultural learning opportunities, or other experiences, which enhance their readiness. Well-prepared HCPs will be better equipped to contribute to relief operations, whereas inadequately prepared HCPs can be a drain on team resources and limit the care and services that can be offered to individuals and communities. ${ }^{22}$

The findings also have relevance for NGOs and other agencies that deploy HCPs in settings of humanitarian crisis. Organizations adopt different strategies to select, prepare, and support clinical staff. ${ }^{23}$ Aspects of moral experience identified in this study are relevant to each of these tasks. Healthcare providers should be selected with skill sets that match operational needs, who exhibit humility and introspective attitudes, and appear to be resilient. Recruitment should aim to identify and exclude $\mathrm{HCPs}$ with unrealistic expectations or overly idealized motivations. This process should help to ensure that project staff are able to address local needs. Pre-departure training presents an important opportunity to encourage $\mathrm{HCPs}$ to evaluate the moral dimensions of this work. Experienced field staff can engage departing HCPs in discussion around these issues.

Two strategies in the induction of new staff to local projects also might yield benefits for HCPs in responding to issues raised by this study. Local team practices could include the linking of new staff members with a seasoned colleague. Such a mentor would be a valuable resource for discussing challenging issues that arise for the newly arrived HCP. This strategy may be particularly valuable in larger teams. Another strategy that teams could consider is the partnering of new HCPs in a particular setting with a local staff member, health worker, or other community representative. The identified person could act as a culturebroker and key informant for the expatriate HCP.

The objective of this study was to examine the moral experience of expatriate HCPs in humanitarian crises. A limitation of the study was the difficulty in maintaining an exclusive focus on humanitarian emergencies and disasters due to natural hazards as most participants had experience in a variety of global health contexts. The moral dimension of healthcare practice in humanitarian work is an important and complex area of inquiry. Further analysis of this topic is needed. In particular, research that incorporates the experi- 
ence and perspectives of local HCPs, as well as beneficiaries of assistance, would broaden and greatly enrich this discussion.

\section{Conclusions}

Expatriate HCPs deliver care and services to individuals and populations affected by humanitarian crises. This work entails significant changes from the context of clinical practice in their home countries. In this qualitative inquiry, five themes were identified regarding the moral experience of HCPs who provide care and services for individuals and populations in humanitarian settings. These findings illuminate key moral dimensions of healthcare practice in humanitarian work. In consequence, the following areas should be considered in personal and organizational preparation for engagement in humanitarian work, and incorpo- rated into support structures in field operations: (1) the examination of motivations and expectations; (2) the relational nature of humanitarian work; (3) attention to steep power imbalances; (4) the need to acknowledge and confront limitations in the ability to assist; and (5) recognition of how organizational forms and structures shape everyday moral experience.

\section{Acknowledgements}

The author is grateful to Leigh Turner and Frank Carnevale for reviewing the analysis and Leigh Turner, Frank Carnevale, and Nicholas King for the helpful comments on this manuscript. The author also is grateful to the research participants for taking part in this study.
References

1. Brennan RJ, Nandy R: Complex humanitarian emergencies: A major global health challenge. Emerg Med J 2001;13:147-156.

2. Toole MJ: Mass population displacement: A global public health challenge. Infect Dis Clin North Am 1995;9:353-365.

3. Sondorp E, Kaiser T, Zwi A: Beyond emergency care: Challenges to health planning in complex emergencies. Trop Med Int Health 2001;6(12):965-970.

4. Banatvala $\mathrm{N}$, Zwi $\mathrm{AB}$ : Public health and humanitarian interventions: Developing the evidence base. BMJ 2000;321:101-105.

5. Pantuliano $S, O^{\prime}$ Callaghan $S$ : The 'protection crisis': A review of field-based strategies for humanitarian protection in Darfur. Overseas Development Institute: London. Humanitarian Policy Group. Discussion Paper, 2006.

6. Bjerneld M, Lindmark G, Diskett P, Garrett MJ: Perceptions of work in humanitarian assistance: Interviews with returning Swedish health professionals. Disaster Manag Response 2004;2:101-108.

7. Petrasek D: Moving Forward on the development of minimum humanitarian standards. Am J Int Law 1998;92(3):557-563.

8. Sphere Project: The Humanitarian Charter and Minimum Standards in Disaster Response. Available at http://www.sphereproject.org. Accessed 04 August 2008.

9. Humanitarian Accountability Partnership-International. Available at http://www.hapinternational.org. Accessed 25 August 2008.

10. Moore J, (ed): Hard Choices: Moral Dilemmas in Humanitarian Intervention. Lanham, Maryland: Rowman and Littlefield Publishers, 1998.

11. International Federation of Red Cross and Red Crescent Societies (IFRC): World Disasters Report 2003. Focus on ethics in aid. Geneva: IFRC, 2003

12. Slim H: Doing the right thing: Relief agencies, moral dilemmas, and moral responsibility in political emergencies and war. Disasters 1997;21(3):244-257.
13. Hunt MR: Ethics beyond borders: How health professionals experience ethics in humanitarian assistance and development work. Dev World Bioetb 2008;8(2):59-69.

14. Fox RC, Goemaere E: They call it "patient selection" in Khayelitsha: The experience of Médecins sans Frontières-South Africa in enrolling patients to receive antiretroviral treatment for HIV/AIDS. Camb $Q$ Healthe Etbics 2006;15:302-312.

15. Michael M, Zwi AB: Oceans of need in the desert: Ethical issues identified while researching humanitarian agency response in Afghanistan. Dev World Bioethics 2002;2(2):109-130.

16. Carnevale FA, Canoui $\mathrm{P}$, Hubert $\mathrm{P}$, et at: The moral experience of parents regarding life-support decisions for their critically-ill children: A preliminary study in France. J Child Health Care 2006;10:69-82.

17. Thorne S: Interpretive Description. Walnut Creek, CA: Left Coast Press, 2008.

18. Thorne S, Reimer Kirkham S, O'Flynn-Magee K: The analytic challenge in interpretive description. Int J Qualitative Methods 2004;3(1):1337-1352

19. Glaser BG, Strauss AL: The Discovery of Grounded Theory: Strategies for Qualitative Research. Chicago, IL: Aldine, 1967.

20. Lincoln YS, Guba EG: Naturalistic Inquiry. Beverly Hills, CA: Sage, 1985.

21. Hilhorst D, Schmiemann N: Humanitarian principles and organizational culrure: Everyday practice in Médecins sans Frontières-Holland. Development in Practice 2002;12(3-4):490-500.

22. Birch M, Miller S: Humanitarian assistance: Standards, skills, training, and experience. BMJ 2005;330:1199-1201

23. Moresky RT, Eliades MJ, Bhimani MA, et al: Preparing international relief workers for healthcare in the field: An evaluation of organizational practices. Prebosp Disaster Med 2001;16(4):257-262. 